\title{
Intoxicación por cianuro secundaria a exposición dérmica e inhalatoria no intencional: Reporte de caso y Revisión de la Literatura.
}

\author{
Jonathan Espinosa ${ }^{1}$, Sebastián Hernández $\mathrm{M}^{2}$, Vivian M. Laguado $\mathrm{C}^{3 *}$, \\ Emiro Nel Valverde $G^{4}$, Daniel Mauricio Núñez $C^{5}$.

\begin{abstract}
${ }^{1}$ Médico Interno, Fundación Santa Fe de Bogotá, Universidad Andes. Bogotá-Colombia. ${ }^{2-3}$ Residente Medicina de Emergencias Universidad el Rosario. Bogotá-Colombia.

${ }^{4-5}$ Especialista en Medicina de Emergencias, Fundación Santa Fe. Bogotá-Colombia.
\end{abstract}

\section{RESUMEN}

Introducción. El Cianuro es un químico de fácil obtención en el mercado utilizado en varias industrias como la producción de insecticidas, fotografía y joyería. Los casos de intoxicación debidos a este compuesto tienen una alta tasa de mortalidad, sobre todo aquellos en los que no se administran de manera oportuna los antídotos. Esto es aplicable tanto a países industrializados como a los países en vías de desarrollo, lugares donde no se cuenta con recursos suficientes para el manejo de estas situaciones medicas complejas, como es el caso de nuestro medio, donde la incidencia de este tipo de intoxicaciones no es clara y puede estar subregistrada, reportándose dentro del grupo de intoxicaciones por sustancias químicas, siendo con intencionalidad suicida la mitad de los casos y la mayoría en hombres.

Objetivo. Conocer y manejar de manera adecuada los casos de intoxicación por cianuro, resulta de gran importancia que en los servicios de urgencias se tenga claridad sobre los aspectos fisiopatológicos y clínicos involucrados y la disponibilidad para uso de sustancias como el nitrito de sodio, el nitrito de amilo, el tiosulfato de sodio y la hidroxicobalamina como parte del tratamiento de estos pacientes, compuestos que no están habitualmente disponibles en nuestro país, siendo el azul de metileno el antídoto de elección por su disponibilidad en nuestro medio.

Metodología. Se trata de un reporte de caso de una paciente con intoxicación por cianuro secundaria a exposición dérmica en la fundación santa fe de Bogotá en 2020 y revisión de la literatura.

Resultados. Reportamos el caso de una paciente de 68 años, quien sufre una exposición involuntaria a cianuro por lo cual ingresa al servicio de urgencias con síntomas inespecíficos secundarios a dicha exposición, con evidencia de acidemia metabólica como principal hallazgo paraclínico. Se documenta la evolución clínica y de la información aportada por el laboratorio y las medidas disponibles utilizadas para su manejo.

Conclusión. Exponemos un caso de intoxicación por cianuro por vías poco comunes en nuestro medio, las medidas utilizadas y las limitaciones que observamos al enfrentarnos a un escenario que potencialmente llevaría a la muerte a la paciente.

Palabras clave: Cianuro; Intoxicación; Antídotos; Dérmica; Inhalatoria.

\section{ABSTRACT}

Cyanide Poisoning Secondary to Unintentional Dermal and Inhalation Exposure: Case Report and Literature Review.

Introduction. Cyanide is a readily available chemical on the market used in various industries such as insecticide production, photography, and jewelry. The cases of poisoning due to this compound have a high mortality rate, especially those in which the antidotes are not administered in a timely manner. This is applicable to both industrialized and developing countries, the last ones, places where there are not enough resources to manage these complex medical situations, as is the case in our environment, where the incidence of this type of poisoning is not clear and it may be underregistered, being reported within the group of intoxications by chemical substances, with half of the cases being by suicidal intention and a greater incidence in men.

Objective. In order to know and adequately manage cases of cyanide poisoning, it is of great importance that the emergency services have clear information about the pathophysiological and clinical aspects involved and the availability for use of substances such as sodium nitrite, nitrite of amyl, sodium thiosulfate and hydroxycobalamin as part of the treatment of these patients, compounds that are not usually available in our country, with methylene blue being the antidote of choice due to its availability in our country.

Methodology. This is a case report of a patient with cyanide poisoning secondary to dermal exposure at the Santa Fe Foundation in Bogotá in 2020 and a review of the literature

Results. We report the case of a 68-year-old woman who suffers an involuntary exposure to cyanide, for which she is admitted to the emergency deparment with nonspecific symptoms secondary to said exposure, in wich we found evidence of metabolic as the main laboratory finding. The clinical evolution and the information provided by the laboratory and the available measures used for its management are documented.

Conclusion. We expose a case of cyanide poisoning by ways taht are not common in our enviorment the measures used and the limitations that we observe when facing to a scenario that would potentially lead to the death of the patient.

Keywords: Cyanide; Poisoning; Antidotes; Dermal; Inhalation.

*Autor de correspondencia Vivian M. Laguado C. vivilaguado@gmail.com

Como citar: Espinosa Jonathan, Hernández Manzanera S, Laguado Castro VM, Valverde Galván EN, Núñez Castro DM. Intoxicación por cianuro secundaria a exposición dérmica e inhalatoria no intencional: Reporte de caso y Revisión de la Literatura. Revista Cuarzo 2020;26(2):30-34.

Recibido: 28 de octubre de 2020 Aceptado: 27 de noviembre de 2020 Publicado: 30 de diciembre de 2020

DOI: $\underline{\text { https://doi.org/10.26752/cuarzo.v26.n2.531 }}$

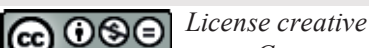




\section{INTRODUCCIÓN}

$\mathrm{C}^{1}$ 1 cianuro es un compuesto químico utilizado en la industria de insecticidas, fotografía y joyería. Es una sustancia de acción rápida utilizada con fines delictivos en varios escenarios. El cianuro causa intoxicación y las personas pueden estar expuestas a dicho efecto mediante diversos tipos de exposiciones entre las que se pueden incluir: incendios, industriales, luego de la administración de medicamentos como nitroprusiato de sodio y alimentarias (1).

En Colombia, la incidencia anual de intoxicaciones por cianuro no es clara. Los datos entregados por el Instituto Nacional de Salud del año 2019 incluyen varias clasificaciones en las cuales podría verse involucrada la utilización de este compuesto y que no hacen referencia específicamente al mismo (medicamentos, plaguicidas, otras sustancias químicas) (2).

En Colombia, datos del Instituto Nacional de Salud (INS) muestran que para la semana epidemiológica 34 del 2019, el $50 \%$ de las muertes relacionadas con intoxicaciones por sustancias químicas tuvieron intencionalidad suicida. El 53\% de estas intoxicaciones ocurrieron en el hogar. Los hombres componen la mayor proporción de afectados y las edades en que más suelen presentarse estos eventos están entre los 12 y 26 años (4).

Por otra parte, las cifras obtenidas en países como Estados Unidos donde en el periodo de 1993 a 2015 se presentaron 3165 exposiciones a cianuro con una mortalidad del $2.5 \%$, siendo la inhalación en casos de incendio la fuente más común (3).

A continuación, presentamos el caso de una paciente que presentó síntomas leves de toxicidad por exposición accidental a cianuro, que tuvo una recuperación posterior completa sin secuelas neurológicas.

\section{REPORTE DE CASO}

Mujer de 68 años colombiana de ocupación ama de casa ingresó a urgencias por cefalea aguda de 10 horas y síntomas de debilidad y malestar general luego de estar en contacto estrecho con fluidos del cuerpo de su hijo intoxicado con cianuro Ingerido por vía oral al intentar realizar maniobras de reanimación mediante respiración boca a boca la cual reportó síntomas gastrointestinales dados por náuseas y tres episodios eméticos de contenido alimentario.

La paciente ingresa al área de reanimación con afecto ansioso, diaforesis, náuseas $\mathrm{y}$ dolor abdominal difuso de intensidad moderada. No tenía antecedentes patológicos de importancia, así como tampoco quirúrgicos ni farmacológicos.

$\mathrm{Al}$ ingreso se monitoriza a la paciente encontrando paciente taquicárdica con frecuencia cardiaca de 110 latidos por minuto, taquipneica con frecuencia respiratoria de 23 respiraciones por minuto, diaforética, hipoxémica con saturación de oxígeno al ambiente de $87 \%$ y cefalea holocraneana tipo pulsátil de intensidad moderada no asociado a trastornos visuales ni déficit neurológico.

Los síntomas presentados se atribuyeron a una intoxicación por cianuro debidas/relacionadas a la exposición dérmica e inhalatoria no intencional. Recibió manejo con oxígeno suplementario a por mascara de no Re inhalación para mantener saturaciones por encima de $90 \%$, hidratación parenteral con cristaloides, lactato de ringer administrándosele bolo inicial de 1000 centímetros cúbicos seguido de infusión de 80 centímetros cúbicos; se tomaron paraclínicos, se encontró acidosis metabólica en gases arteriales y un anión gap elevado con hiperlactatemia, hiponatremia moderada sin otros trastornos hidroelectrolíticos así como función renal y hepática normal.

Tabla 1: Paraclínicos

\begin{tabular}{|c|c|c|c|c|c|}
\hline Parámetro & Ingreso & $\begin{array}{l}4 \text { horas } \\
\text { del } \\
\text { Ingreso }\end{array}$ & $\begin{array}{c}11 \\
\text { horas } \\
\text { del } \\
\text { ingreso } \\
\end{array}$ & $\begin{array}{c}20 \\
\text { horas } \\
\text { del } \\
\text { ingreso } \\
\end{array}$ & $\begin{array}{c}\text { Valor } \\
\text { Normal }\end{array}$ \\
\hline $\begin{array}{r}\text { Transaminasa } \\
\text { Glutámico } \\
\text { oxalacetica } \\
(U / L)\end{array}$ & 30 & 26 & & & $0.0-35.0$ \\
\hline $\begin{array}{r}\text { Transaminasa } \\
\text { glutámico- } \\
\text { pirúvica }(\mathrm{U} / \mathrm{L})\end{array}$ & 41 & & & & $0.0-35.0$ \\
\hline$I N R$ & 1.11 & & & & \\
\hline $\begin{array}{r}\text { Magnesio } \\
(\mathrm{mg} / \mathrm{dl})\end{array}$ & 1.56 & 1.54 & & & $1.90-2.50$ \\
\hline Calcio (mg/dl) & 9.8 & & & & $8.80-10.6$ \\
\hline Cloro $(m E q / L)$ & 100 & 101 & & & $98-107$ \\
\hline $\begin{array}{r}\text { Potasio } \\
(m E q / L)\end{array}$ & 4.01 & 4.36 & & 4 & $3.50-5.10$ \\
\hline Sodio $(m E q / L)$ & 129 & 131 & & 141 & $136-146$ \\
\hline $\begin{array}{r}\text { Troponina } \\
(\mathrm{mg} / \mathrm{L})\end{array}$ & 6.5 & & & & $0.55-1.02$ \\
\hline $\begin{array}{r}\text { Creatinina } \\
(\mathrm{mgl} / \mathrm{dl})\end{array}$ & 0.69 & 0.74 & & 0.83 & $0.55-1.02$ \\
\hline$B U N(m g / d l)$ & 12 & 10 & & 11 & jul-25 \\
\hline Amilasa $(U / L)$ & 75 & & & & $29-103$ \\
\hline $\begin{array}{r}\text { Plaquetas } \\
(10 * 3 / u l)\end{array}$ & 186 & 243 & & 193 & $150-450$ \\
\hline $\begin{array}{r}\text { Hemoglobina } \\
(\mathrm{g} / \mathrm{dL})\end{array}$ & 10.6 & 13.1 & & 11.9 & dic-16 \\
\hline$P h$ & 7.59 & 7.49 & 7.43 & 7.46 & $7.40-7.46$ \\
\hline pO2 (mmhg) & 143 & 171 & 100 & 74 & $60-100$ \\
\hline Pco2 (mmhg) & 13 & 21 & 25 & 24 & $28-32$ \\
\hline $\begin{array}{r}\mathrm{HCO3} \\
(\mathrm{mmol} / \mathrm{L}) \\
\end{array}$ & 12.5 & 16 & 16.6 & 17.1 & $18-22$ \\
\hline $\begin{array}{r}\text { Fosfatasa } \\
\text { alcalina }(U / L)\end{array}$ & 76 & 68 & & & $30.0-120.0$ \\
\hline
\end{tabular}

Ante la exposición y clínica sugerente de intoxicación por cianuro se contactó al Ministerio de Salud y la línea toxicológica nacional obteniendo tiosulfato de sodio al $20 \%$ y se administrándosele como antídoto específico 2 gramos en 30 minutos. Posterior a lo cual se observó resolución de acidosis e hiperlactatemia, así como mejoría del patrón respiratorio. 
Luego de una hospitalización de 20 horas en el área de observación de urgencia la paciente tuvo resolución de la cefalea y la pulso-oximetría, así como corrección paraclínica dado por depuración del lactato y corrección de la hiponatremia y la hipocalemia pudiendo así egresar del hospital sin eventualidades.

\section{DISCUSIÓN}

Actualmente la exposición a cianuro es una causa poco común de intoxicación en nuestro medio, aún más infrecuentes son las intoxicaciones por la vía transdérmica. Sin embargo, para el año 2000 el envenenamiento por esta sustancia constituyó la primera causa de suicidio en Bogotá (4). Aunque las vías de administración más comunes es la oral e inhalación. La piel también puede ser una ruta potencialmente importante de exposición en el manejo y uso de cianuros en procesos como galvanoplastia, extracción de minerales, agricultura y operaciones de fumigación (5). Si además se tienen en cuenta los casos reportados de intoxicación por medio de medicamentos que contienen este compuesto y algunos alimentos (6). El cianuro de hidrógeno, cianuro de sodio y cianuro de potasio son las formas de sales en las que se encuentra este compuesto en orden de toxicidad percutánea (7). Se ha evidenciado en modelos animales que dependiendo el estado de la piel (intacta, húmeda o con soluciones de continuidad) se puede llegar a una LD50 (dosis letal para el $50 \%$ de la población expuesta) con soluciones de 6.9 y 2.43 miligramos por kilo de $\mathrm{HCN}$ en piel intacta o lesionada, respectivamente. En otras palabras, una persona de 70 kilogramos de peso que exponga su piel a 0.5 gramos de cianuro de hidrógeno puede fallecer (8).

En Colombia, las sales de cianuro son de venta libre, el costo aproximado de un kilogramo de cianuro de potasio es de 25.000 pesos, es soluble en glicerina, alcohol y agua. Las manifestaciones clínicas previas al deceso en los animales expuestos a las dosis letales previamente mencionadas incluyen temblores, espasmos retro-cólicos, convulsiones, patrones de respiración anormales y postración.

A continuación, reportamos el caso de una paciente con síntomas asociados a dicha exposición indirecta, quien resolvió la sintomatología posterior al inicio de medidas de soporte y finalmente a la aplicación de tiosulfato de sodio como antídoto.

\section{Fisiopatología.}

El cianuro es un químico de acción rápida y altamente tóxico que genera intoxicaciones potencialmente fatales. Consiste en una molécula de triple unión al nitrógeno (4). Estos compuestos contienen un grupo ciano que se expresa como CN (8). Es altamente citotóxico e interactúa rápidamente con el hierro trivalente $(\mathrm{Fe} 3+)$ de la citocromo oxidasa $\mathrm{A} 3$, lo que produce una parálisis de la cadena transportadora de electrones en la mitocondria, la producción de ATP y la glicólisis aerobia. Todo esto da como resultado: producción elevada de ácido láctico, disfunción tisular, choque y muerte (9). El cianuro se puede encontrar en varias formas tales como sales (cianuro inorgánico o en su forma aniónica como CN -) (1).
El cianuro es absorbido rápidamente por el tracto respiratorio y las membranas mucosas, al igual que en el tracto gastrointestinal y la piel (10). El inicio de los síntomas y su severidad se asocian a la vía de exposición: la endovenosa y la inhalatoria son las que producen un inicio más rápido y de mayor severidad en comparación con la vía oral y transdérmica. El cianuro actúa mediante la unión reversible a los iones de hierro del citocromo $\mathrm{C}$ oxidasa mitocondrial de forma que impide la respiración celular al bloquear la reducción de oxígeno a agua, para finalmente inhibir la fosforilación oxidativa y causar hipoxia celular y depleción de ATP, que lleva a la acidosis metabólica (11).

Dentro de las causas de intoxicación. por cianuro tenemos como la causa más común en países industrializados la exposición por fuego por incendios domésticos debido a la liberación de carbón y nitrógeno durante la combustión de productos como (poliestireno, resinas, madera, poliuretano, poliacrilonitrilos y materiales sintéticos) (1). A nivel industrial tenemos fuentes de cianuro en la producción de oro, minería, fotografía, removedores de uñas, manufactura de plástico, pesticidas y fumigantes (2).

\section{Manifestaciones Clínicas.}

Los pacientes pueden presentar síntomas rápidamente (hasta 1 minuto después de la exposición). Si es inhalado el paciente puede referir olor a almendras amargas. Las manifestaciones se pueden dividir en tempranas y tardías (12). Entre las manifestaciones neurológicas tempranas encontramos: cefalea, mareo, confusión y midriasis, con efectos tardíos como convulsiones y coma. A nivel respiratorio y cardiovascular pueden presentarse taquipnea y taquicardia. En cuanto a las manifestaciones tardías se pueden dar: apnea, hipotensión y arritmias cardiacas. La bradicardia y la hipotensión son patognomónicos, y a pesar de la hipoxia tisular, los pacientes no presentarán cianosis, al contrario, tendrán una tonalidad cereza secundaria al exceso de oxígeno en el torrente sanguíneo (13).

\section{Tratamiento.}

Debido a los síntomas inespecíficos y resultados de laboratorio confirmatorios demorados, y en ocasiones servicios de urgencias ausentes. El diagnóstico debe basarse en la sospecha clínica (teniendo en cuenta el contexto del paciente) y los hallazgos iniciales entre los que se encuentran: colapso súbito de un trabajador de laboratorio o industrial, víctima de incendio en coma o con acidosis metabólica (14), intento suicida en coma o con acidosis inexplicada, ingestión de removedor de uñas, ingestión de semillas de Prunus spp, alteración del estado de conciencia, acidosis metabólica y taquifilaxia con el uso de nitroprusiato(15).

A su ingreso, debe darse soporte, control de la vía aérea, ventilación, oxígeno al $100 \%$, infusión de cristaloides y de ser necesario vasopresores, administración de bicarbonato de sodio titulado según gasometría $(15,16)$. Concomitantemente según la disponibilidad se debe administrar un antídoto para lograr la eliminación. 
Los antídotos se enfocan en ligar el cianuro libre usando moléculas que contienen cobalto y compuestos de nitrito y/o aumentar la eliminación del cianuro como tiocianato usando tiosulfato de sodio u otros donantes de azufre como el sulfanegen $(11,17)$.

Dentro de los medicamentos usados para controlar los efectos deletéreos del cianuro se incluyen, el azul de metileno y el tiosulfato de sodio.

El azul de metileno es un pigmento que posee propiedades redox al entrar en la sangre, y que es inmediatamente reducido a azul de leucometileno por muchos de los agentes reductores (NADPH, glutatión reducido), posteriormente puede ser nuevamente re-oxidado a azul de metileno por moléculas con mayor potencial redox (Oxígeno o metalo- compuestos), donando electrones e iniciando un nuevo ciclo de reducción. Difundiendo dentro de la célula (citoplasma y mitocondria), en sus dos formas, ejerce su efecto según el estado redox del ambiente inmediato y ante sus propiedades bajo su forma oxidada/reducida tan variable, es difícil predecir qué proceso está implicado en la protección contra la intoxicación por cianuro (17).

Por lo anterior se consideran 2 posibles vías que explican el efecto protector del azul de metileno en la intoxicación por cianuro. El primero: en su forma oxidada actúa como captador de electrones y puede llevar a metahemoglobinemia, es decir como consumidor de cianuro. Segundo, en su forma reducida, el azul de leucometileno interactúa directamente con la cadena de electrones mitocondrial, donando electrones a los complejos I y III y/o permitiendo la restauración parcial del ciclo de Krebs (17).

El tiosulfato de sodio actúa catalizando la formación de tiocianato del cianuro al donar un grupo sulfidrilo a la enzima rodenasa, por lo cual se considera un antídoto conversor de metabolitos a compuestos menos tóxicos que pueden ser excretados (18).

Otro mecanismo para la eliminación y control de los síntomas asociados a la intoxicación por cianuro es la unión específica de la hidroxicobalamina $(18,19)$. La hidroxicobalamina es una metaloproteína con un átomo de cobalto central que se une al cianuro formando cianocobalamina, la cual es eliminada en la orina o disminuyendo la concentración libre de cianuro, permitiendo la desintoxicación por la rodenasa (15).

Cabe recalcar la existencia de kits para tratamiento de pacientes intoxicados con cianuro, que incluyen nitrito de amilo, nitrito de sodio y tiosulfato de sodio, los cuales, usados en combinación, han demostrado mayor beneficio que su uso por separado (15).

No debemos olvidar que ante la contaminación por vía cutánea durante el manejo inicial se debe hacer una descontaminación $\neg$ para retirar el cianuro persistentemente en contacto con el paciente y también para proteger al personal de la salud tratante. Este proceso incluye retirar la ropa y lavar la piel. Y ante la ingestión de cianuro se requiere lavado gástrico e infusión de carbón activado (15).

En Colombia, el antídoto disponible en el servicio de urgencias es el azul de metileno cuya indicación es el uso inmediato frente a la sospecha de intoxicación por cianuro. En cuanto al tiosulfato de sodio no es un medicamento disponible en las farmacias de los servicios de emergencias, por lo que, como en este caso, se debe diligenciar la solicitud de este a la Secretaría de Salud para su entrega, que es un factor modificable de demora en la atención de los pacientes.

\section{CONCLUSIONES}

La intoxicación por cianuro secundaria a exposición transdérmica e inhalatoria no intencional es poco frecuente a nivel mundial por lo que se considera que una alta sospecha y experticia diagnóstica del médico a cargo a través de la clínica presentada por el paciente es crucial.

En la intoxicación por cianuro es muy importante la concentración y la vía de administración; a dosis bajas. Los síntomas generalmente son leves y los pacientes tienen un buen pronóstico, pero aquellos con exposición a dosis altas pueden tener desenlaces fatales. En relación con las vías de administración, la vía transdérmica generalmente conlleva a efectos menores y mejores desenlaces además de ser un tipo de intoxicación poco frecuente.

Con este reporte de caso queremos plasmar una vía de intoxicación con cianuro poco frecuente, que debemos tener presente ya que dependiendo de la dosis podría llevar a la muerte. De aquí también la importancia de tener disponibles los antídotos necesarios para manejar este tipo de intoxicaciones en los servicios de urgencias.

Conflicto de Interés: Los autores no declaran conflicto de interés.

\section{REFERENCIAS}

1. Parker-Cote JL, Rizer J, Vakkalanka JP, Rege SV, Holstege $\mathrm{CP}$. Challenges in the diagnosis of acute cyanide poisoning. Clinical toxicology. 2018 Jul 3;56(7):609-17.

2. Instituto Nacional De Salud (INS). Boletín Epidemiológico Semanal: Comportamiento de la Vigilancia de Intoxicaciones por Sustancias, Ministerio de Salud (internet) (Consultado 31 mayo 2020). Disponible en: https://www.ins.gov.co/buscadoreventos/BoletinEpidemiologico/2020 Boletin epidemiolog ico semana 6.pdf

3. Hamad E, Babu K, Bebarta VS. Case files of the University of Massachusetts toxicology fellowship: does this smoke inhalation victim require treatment with cyanide antidote?. Journal of Medical Toxicology. 2016 Jun;12(2):192-8. 
4. Iregui MC, Contreras CM, Restrepo MU. Envenenamiento por cianuro. Revista Colombiana de Psiquiatría. 2002;31(4):271-5.

5. Kales SN, Christiani DC. Acute chemical emergencies. New England Journal of Medicine. 2004 Feb 19;350(8):800-8.

6. Suchard JR, Wallace KL, Gerkin RD. Acute cyanide toxicity caused by apricot kernel ingestion. Annals of emergency medicine. 1998 Dec 1;32(6):742-4.

7. Curry SC, Spyres MB. Cyanide: hydrogen cyanide, inorganic cyanide salts, and nitriles. Critical care toxicology. Diagnosis and management of the critically poisoned patient. Elsevier-Mosby. 2005;987:C1.

8. Ballantyne B. Acute percutaneous systemic toxicity of cyanides. Journal of Toxicology: Cutaneous and Ocular Toxicology. 1994 Jan 1;13(3):249-62.

9. Thomas TA, Brooks JW. Accidental cyanide poisoning. Anaesthesia. 1970 Mar;25(1):110-4.

10. Hall AH, Rumack BH. Clinical toxicology of cyanide. Annals of Emergency Medicine. 1986 Sep 1;15(9):1067-74.

11. Hsiao PJ, Chang CF, Chiu CC, Chan JS, Chiang WF, Wu $\mathrm{CC}$, Lin SH, Chen JS. High anion gap metabolic acidosis after a suicide attempt with cyanide: the rebirth of cyanide poisoning. Internal medicine. 2015;54(15):1901-4.

12. Graham J, Traylor J. Cyanide toxicity.

13. Holstege CP, Forrester JD, Borek HA, Lawrence DT. A case of cyanide poisoning and the use of arterial blood gas analysis to direct therapy. Hospital Practice. 2010 Nov $1 ; 38(4): 69-74$.

14. Holstege CP, Cyanide and hydrogen sulfide. 2014

15. Brivet F, Delfraissy JF, Duche M, Bertrand P, Dormont J. Acute cyanide poisoning: recovery with non-specific supportive therapy. Intensive care medicine. 1983 Jan;9(1):33-5.

16. Haouzi P, Gueguinou M, Sonobe T, Judenherc-Haouzi A, Tubbs N, Trebak M, Cheung J, Bouillaud F. Revisiting the physiological effects of methylene blue as a treatment of cyanide intoxication. Clinical Toxicology. 2018 Sep 2;56(9):828-40.

17. Chacko B, Peter JV. Antidotes in Poisoning. Indian journal of critical care medicine: peer-reviewed, official publication of Indian Society of Critical Care Medicine. 2019 Dec;23(Suppl 4):S241.

18. Hall AH, Rumack BH. Hydroxycobalamin/sodium thiosulfate as a cyanide antidote. The Journal of emergency medicine. 1987 Jan 1;5(2):115-21. 